\title{
Can gravity waves significantly impact PSC occurrence in the Antarctic?
}

\author{
A. J. McDonald, S. E. George, and R. M. Woollands \\ Department of Physics and Astronomy, University of Canterbury, Private Bag 4800, Christchurch, New Zealand
}

Received: 11 December 2008 - Published in Atmos. Chem. Phys. Discuss.: 3 February 2009

Revised: 14 October 2009 - Accepted: 25 October 2009 - Published: 23 November 2009

\begin{abstract}
A combination of POAM III aerosol extinction and CHAMP RO temperature measurements are used to examine the role of atmospheric gravity waves in the formation of Antarctic Polar Stratospheric Clouds (PSCs). POAM III aerosol extinction observations and quality flag information are used to identify Polar Stratospheric Clouds using an unsupervised clustering algorithm.

A PSC proxy, derived by thresholding Met Office temperature analyses with the PSC Type Ia formation temperature $\left(T_{\mathrm{NAT}}\right)$, shows general agreement with the results of the POAM III analysis. However, in June the POAM III observations of PSC are more abundant than expected from temperature threshold crossings in five out of the eight years examined. In addition, September and October PSC identified using temperature thresholding is often significantly higher than that derived from POAM III; this observation probably being due to dehydration and denitrification. Comparison of the Met Office temperature analyses with corresponding CHAMP observations also suggests a small warm bias in the Met Office data in June. However, this bias cannot fully explain the differences observed.

Analysis of CHAMP data indicates that temperature perturbations associated with gravity waves may partially explain the enhanced PSC incidence observed in June (relative to the Met Office analyses). For this month, approximately $40 \%$ of the temperature threshold crossings observed using CHAMP RO data are associated with small-scale perturbations. Examination of the distribution of temperatures relative to $T_{\mathrm{NAT}}$ shows a large proportion of June data to be close to this threshold, potentially enhancing the importance of gravity wave induced temperature perturbations. Inspection of the longitudinal structure of PSC occurrence in June 2005 also shows that regions of enhancement are geographically
\end{abstract}

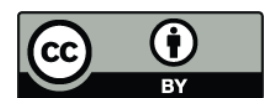

Correspondence to: A. J. McDonald (adrian.mcdonald@canterbury.ac.nz) associated with the Antarctic Peninsula; a known mountain wave "hotspot". The latitudinal variation of POAM III observations means that we only observe this region in June-July, and thus the true pattern of enhanced PSC production may continue operating into later months.

The analysis has shown that early in the Antarctic winter stratospheric background temperatures are close to the $T_{\mathrm{NAT}}$ threshold (and PSC formation), and are thus sensitive to temperature perturbations associated with mountain wave activity near the Antarctic peninsula (40\% of PSC formation). Later in the season, and at latitudes away from the peninsula, temperature perturbations associated with gravity waves contribute to about $15 \%$ of the observed PSC (a value which corresponds well to several previous studies). This lower value is likely to be due to colder background temperatures already achieving the $T_{\mathrm{NAT}}$ threshold unaided. Additionally, there is a reduction in the magnitude of gravity waves perturbations observed as POAM III samples poleward of the peninsula.

\section{Introduction}

The role of PSCs in polar ozone depletion was first identified over twenty years ago (Solomon et al., 1986). Heterogeneous chemical reactions on PSC particles are the central process in chlorine activation and consequent ozone depletion. In addition to the activation of chlorine from reservoirs of $\mathrm{HCl}$ and $\mathrm{ClONO}_{2}$, PSCs can also remove $\mathrm{NO}_{\mathrm{y}}$ (i.e. the total odd nitrogen) from the lower stratosphere by incorporation of $\mathrm{HNO}_{3}$ from the gas phase and subsequent sedimentation. This denitrification leads to a slower conversion of active chlorine back to the reservoir species $\mathrm{ClONO}_{2}$.

In contrast to the Northern Hemisphere, very low stratospheric temperatures in the Antarctic winter generally lead to an abundance of PSCs in the lower stratosphere (Poole and Pitts, 1994). Three categories of PSCs are generally recognized: Type Ia, composed of solid phase nitric acid trihydrate

Published by Copernicus Publications on behalf of the European Geosciences Union. 
(NAT); Type Ib, composed of supercooled ternary solution (STS, a liquid mixture of $\mathrm{H}_{2} \mathrm{O}, \mathrm{HNO}_{3}$, and $\mathrm{H}_{2} \mathrm{SO}_{4}$ ); and Type II, composed primarily of ice (see Lowe and MacKenzie, 2008, and references therein). These three categories of PSC form at different temperatures in the stratosphere. In particular, the NAT PSC formation temperature $\left(T_{\mathrm{NAT}}\right)$ is dependent on pressure, nitric acid and water vapour concentrations (Hanson and Mauersberger, 1988) and is significantly warmer than the ice frost point $\left(T_{\mathrm{ICE}}\right)$. The STS formation temperature $\left(T_{\mathrm{STS}}\right)$ occurs approximately $3.5 \mathrm{~K}$ below the value of $T_{\mathrm{NAT}}$ (Carslaw et al., 1994) and the ice frost point temperature can be derived by using the formulation described in Marti and Mauersberger (1993). Temperatures are cool enough in the Antarctic stratosphere for all three types of clouds to be present during the winter. The present study examines the potential contribution of internal gravity waves to PSC formation in the Antarctic stratosphere. In the Arctic the temperature perturbations associated with internal gravity waves, particularly mountain waves, have been shown to be responsible for the formation of a significant proportion of PSCs (Carslaw et al., 1998; Höpfner et al., 2001). However, the observed relationship between Antarctic PSC formation and low background temperatures has led to less focus on wave induced PSC formation processes in the Antarctic.

Recent studies have indicated that the temperature perturbations related to these waves may have a more significant effect on Antarctic PSC occurrence and have a potentially important role in ozone depletion (Shibata et al., 2003; Höpfner et al., 2006; Innis and Klekociuk, 2006; Eckermann et al., 2009). Such effects may be particularly significant in early winter where temperatures hover close to PSC formation temperature thresholds. Analysis by Höpfner et al. (2006) has identified a significant role of mountain waves in the formation of PSC and subsequent denitrification in the Antarctic. Höpfner et al. (2006) described spaceborne infrared measurements of a vortex-wide area of NAT PSC around Antarctica using the Michelson Interferometer for Passive Atmospheric Sounding (MIPAS) instrument. Simulations for the period 10-12 June 2003 indicate the observations are likely to be associated with heterogeneous nucleation on ice in the cooling phases of large-amplitude stratospheric mountain waves over the Antarctic Peninsula. The process operating here is the same mountain wave NAT formation model originally proposed by Carslaw et al. (1998) for the Arctic winter stratosphere. Recent work by Eckermann et al. (2009) examined the same MIPAS satellite observations over Antarctica with additional Atmospheric Infrared Sounder (AIRS) data. Their study showed that large mountain wave temperature perturbations produced NAT which is advected to form a circumpolar NAT outbreak which concurs with Höpfner et al. (2006).

Other studies have also examined the role of gravity waves on PSC formation. For example, a ground-based lidar study by Shibata et al. (2003) indicated that Type II PSCs occurred when stratospheric temperatures dropped below $T_{\mathrm{ICE}}$ because of perturbations associated with inertia gravity waves. Another lidar study by Innis and Klekociuk (2006) also estimated the influence of gravity waves on PSC occurrence. They concluded that during the "PSC season" when the background temperature was close enough to the NAT formation temperature $\left(T_{\mathrm{NAT}}\right)$, derived in Hanson and Mauersberger (1988), gravity wave perturbations influence PSC formation approximately $15 \%$ of the time. Interestingly, this value is similar to the findings of Felton et al. (2007) who concluded Type Ia enhanced PSCs, which are likely to be NAT crystals with unusually large lidar backscattering ratios which appear down wind of mountain wave-induced ice clouds (Tsias et al., 1999), make up $11 \%$ of the clouds observed during the SAGE III Ozone Loss and Validation Experiment (SOLVE) campaign in winter 2000 in the Northern Hemisphere. However, the warmer mean temperature in the Arctic means that this is likely to be coincidental. It is also worthy of mention that Innis and Klekociuk (2006) found a clear relationship between planetary wave temperature perturbations and PSC occurrence on the edge of the Antarctic continent. Recent analysis using data from CALIPSO (a space-borne lidar) has also mentioned the potential importance of gravity waves on PSC formation (Pitts et al., 2007; Noel et al., 2008). However, enhanced PSC formation has also been suggested to be related to synoptic-scale motions (Teitelbaum et al., 2001; Wang et al., 2008).

Previous work has shown that NAT PSC formation may be implicitly linked to gravity wave temperature perturbations. In particular, homogeneous NAT nucleation does not occur directly on temperatures crossing $T_{\mathrm{NAT}}$. Rather, average temperatures need to be below the $T_{\mathrm{NAT}}$ threshold for a period greater than a few days for nucleation to occur (Peter, 1997). Carslaw et al. (1994) discusses NAT formation and indicates that NAT may form by heterogeneous nucleation on preexisting ice particles which may form because of large temperature perturbations produced by mountain waves. Work by Fueglistaler et al. (2002) suggests that early season PSC production associated with Type Ia or Ia enhanced clouds, produced using the mechanism proposed in Carslaw et al. (1994), can potentially be extremely important in denitrification due to their role in the formation of extremely large NAT particles if number densities within the clouds are high. However, recent work has additionally identified a need for NAT nucleation mechanisms which are independent of the existence of ice particles, only requiring temperatures below the existence temperature for NAT which is significantly easier (approximately $7-8 \mathrm{~K}$ warmer than $T_{\mathrm{ICE}}$ ) to meet than the demand for pre-existing ice particles.

Voigt et al. (2005) detailed measurements where the conditions of particle formation are well enough constrained to conclude that a PSC is observed in air parcels that spent less than a day (approximately $18 \mathrm{~h}$ ) at temperatures no lower than $3 \mathrm{~K}$ below $T_{\mathrm{NAT}}$. Hitchman et al. (2003) also suggests that a formation mechanism for Type Ia PSC must exist 
without the requirement for temperatures significantly below $T_{\mathrm{NAT}}$. Their study also highlights the potential importance of non-orographic gravity waves on PSC formation in the Arctic. Pagan et al. (2004) conclude that Type Ia PSCs can nucleate in relatively warm synoptic-scale temperature fields and regions of strong mountain-wave activity with sufficient cooling to produce ice nuclei are not required. In addition, Irie et al. (2004) used ILAS trace gas and aerosol extinction measurements, AVHRR stratospheric ice cloud measurements, and a microphysical box model to investigate processes leading to denitrification in the Arctic vortex in February 1997. Their analysis suggest that sedimentation of NAD or NAT particles formed through NAD freezing on aerosol surfaces can cause significant denitrification in the Arctic vortex. Their analysis combined with the results in Pagan et al. (2004) seem to confirm that ice particle surfaces are not a prerequisite for the formation of nitric acid hydrate PSCs. Review of these and other studies detailed in the World Meteorological Organization (2007) report indicates increasing evidence that processes occur above the ice frost point. However, the report indicates that whether these processes are applicable in the Antarctic requires investigation.

This study examines the relationship between PSC occurrence observed using POAM III observations and the occurrence expected based on Met Office analyses and CHAMP radio occultation measurements. The addition of high vertical resolution CHAMP temperature observations provides the possibility of identifying periods and spatial regions where PSC formation associated with gravity wave induced temperature perturbations may occur. Though it should be noted that the CHAMP instrument, in a similar manner to all limb viewing instruments, can only observe a portion of the gravity wave spectrum. Comparison of the observations from Met Office analyses and CHAMP observations also allows the sensitivity of PSC occurrence to temperature biases in Met Office analyses to be examined. In order to examine the importance of temperature and the impact of gravity wave temperature perturbations we determine the probability that a region is below various threshold temperatures relative to the frequency of PSC observations made with POAM III. This method has previously been used by many authors, including Poole and Pitts (1994) and Saitoh et al. (2006).

In this study, Section 2 describes the POAM III and CHAMP RO observations and also details a new unsupervised clustering algorithm which is applied to POAM III extinction measurements to identify PSC. Comparisons of the observed probability of PSC occurrence relative to those based on the occurrence of temperatures below the $T_{\mathrm{NAT}}$, $T_{\mathrm{STS}}$ and $T_{\mathrm{ICE}}$ thresholds derived from Met Office analyses and CHAMP RO data are detailed in Sect. 3 and the interpretation of these results is discussed in Sect. 4.

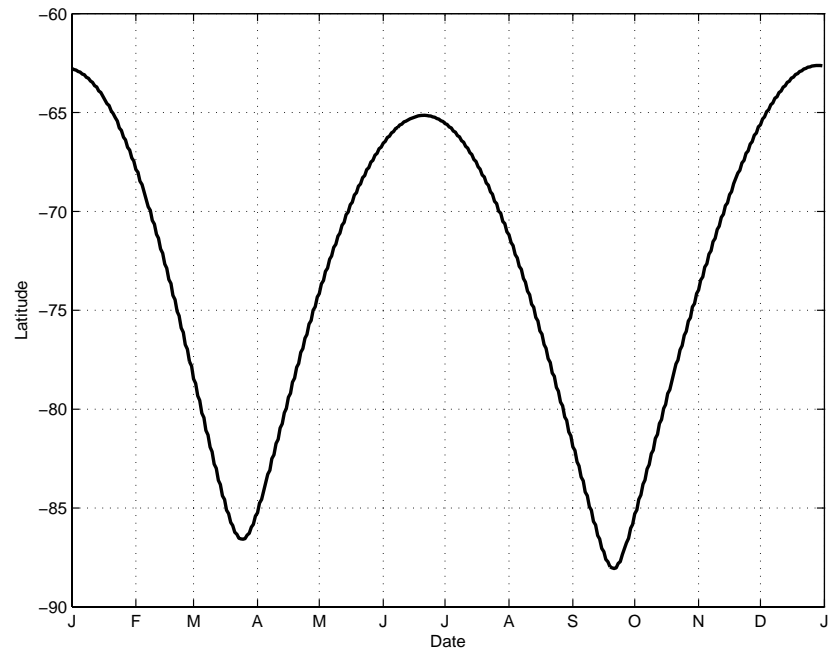

Fig. 1. Seasonal variation of observation latitude measured by POAM III.

\section{Dataset and methodology}

\subsection{POAM III observations and the PSC identification algorithm}

The POAM III instrument made observations of the upper troposphere and stratosphere between its launch on the Satellite Pour l'Observation de la Terre (SPOT) 4 satellite in March 1998 and the end of 2005. POAM measures water vapor, aerosol extinction, ozone, and nitrogen dioxide using the solar occultation limb sounding technique (Lucke et al., 1999). The high inclination of the SPOT 4 satellite allows occultations to be performed in both the northern and southern polar regions. It should be noted that the POAM III sampling volume is about $200 \mathrm{~km}$ long, $30 \mathrm{~km}$ wide, and $1 \mathrm{~km}$ thick and thus this data set will not capture very small-scale features. The POAM III instrument provides 12 to 14 profiles per day in each hemisphere around a circle of latitude. The latitude of the sampled region varies throughout the year and varies from roughly $63^{\circ}$ to $88^{\circ}$ in the Southern Hemisphere (see Fig. 1). This latitudinal variation of the solar occultation satellite observations implies an inherent sampling issue when attempting to separate the impact of latitudinal and temporal variations in this dataset. For example, Pitts et al. (2007) has shown that sampling CALIPSO data at SAM II latitudes moves the maximum PSC occurrence date from early August to September due to the SAM II sampling being at the highest latitude sampled. The fact that both SAM II and POAM III have similar sampling patterns means that issues associated with sampling bias need to be considered. Research completed using POAM III observations has focused on examinations of the spatial and temporal variability of stratospheric ozone, aerosols, polar stratospheric clouds, and polar mesospheric clouds. 
In this work, PSC occurrence is derived from POAM III extinction measurements at $1.018 \mu \mathrm{m}$ and the ratio of the 1.018 to the $0.603 \mu \mathrm{m}$ extinction channels using a $\mathrm{k}$-means unsupervised clustering algorithm described in subsequent paragraphs. Jakob and Tselioudis (2003) have previously utilized a simple clustering algorithm for cloud regime identification in the Tropical Western Pacific region and recent work by Felton et al. (2007) has applied a clustering algorithm to lidar observations of PSC. We use a similar method to that indicated in Jakob and Tselioudis (2003) to separate different types of PSC. Our algorithm can be used to identify both Type Ia and Ib aerosols using the methodology described in Strawa et al. (2002) except that the separation is based on an unsupervised clustering algorithm. However, in this study we do not differentiate between Type Ia and Ib PSC. The separation into different types will be left to a future study. It should be noted that this method provides similar PSC identification ability to algorithms described by Fromm et al. (2003).

The statistical method of cluster analysis is used to separate background aerosol extinctions from those associated with PSCs. As its name suggests, cluster analysis searches for possible "clusters" in a data set by evaluating some measure of distance between individual data points (in this study we use Euclidean distance in extinction ratio/extinction space to separate PSC and non-PSC observations and then an angular measure can be used to separate PSC Type Ia and Ib but is not utilized in this study). It should be noted that to ensure that neither the extinction at $1.018 \mu \mathrm{m}$ or the ratio of 1.018 to the $0.603 \mu \mathrm{m}$ extinction channels dominate in the clustering procedure the values are normalized. Note that in this case a "data point" is an individual set of measurements of aerosol extinction from POAM III at a specific altitude and geographical location. The k-means clustering algorithm used in this study, iteratively searches for a predefined number $(\mathrm{k})$ of clusters, two in this case, using the following scheme:

1. k elements of the data set of size $\mathrm{N}$ are selected at random as distinct cluster members;

2. each of the remaining N-k elements are assigned to the cluster with the nearest centroid (based on Euclidean distance in extinction ratio/extinction) whereby after each assignment the centroid of the cluster is recalculated; and

3. after all elements have been assigned the centroids found in step 2 are used as new seed points and the algorithm is iterated.

Normally fewer than ten iterations are sufficient for the convergence of the algorithm. Note that the random selection of data points indicated in the algorithm does not work on observations from the Arctic because of the small number of observations which include PSC. This is because it becomes difficult to identify physically meaningful clusters initially at random. Thus, some apriori knowledge to select likely PSCs would be required. For example, a methodology similar to Fromm et al. (2003) could be used.

It should be noted at this point that other studies (Fromm et al., 1997, 2003) have indicated that the high extinctions associated with Type II PSC can cause the termination or commencement of occultation events at anomalously high altitudes. This is caused by the PSC having large opacity and/or size preventing POAM III from measuring lower. Thus, the extinction measurements are most useful for identifying Type I PSCs and will undersample Type II PSCs. To reduce this bias we then utilize quality flags, often referred to as ZMIN flags, to identify profiles with anomalously high commencement of occultation events and include these in the statistics shown later.

\subsection{CHAMP RO and Met Office observations}

This study also employs data from the radio occultation (RO) experiment onboard the CHAMP satellite available from 2002 to 2005 . In this experiment GPS radio signal bending due to atmospheric refractivity is measured to determine profiles of atmospheric parameters. The data was processed and provided by the GeoForschungsZentrum (GFZ) Potsdam. Version 005 were used for this study. Specific information on the processing method used is detailed in Wickert et al. (2004). The CHAMP satellite has an inclination of $87^{\circ}$ and therefore the data distribution over the globe is nearly uniform. Inversion of bending angle information allows the instrument to produce approximately $150-200$ profiles of dry temperature each day over the globe. The dry temperature is the temperature that results from an inversion process that assumes that the radio refractive index variation observed by radio occultation satellites is related to temperature variations only and this is a very good assumption in the dry stratosphere. Dry temperature therefore represents the true atmospheric temperature very accurately in this region.

From Fresnel diffraction theory it can be shown that the profiles have a true vertical resolution of approximately $1.4 \mathrm{~km}$ in the stratosphere. However, the data is over-sampled and provided by the GFZ at a vertical spacing of $200 \mathrm{~m}$. The horizontal resolution of an occultation is $200-400 \mathrm{~km}$ along the line of sight (LOS) and on the order of $1-3 \mathrm{~km}$ across the LOS. A large number of studies have described the quality of these temperature measurements. In particular, comparisons of CHAMP RO measurements with analyses and radiosonde observations display excellent agreement (Wickert et al., 2004; Gobiet et al., 2005; Parrondo et al., 2007; Wang and Lin, 2007). For example, Wickert et al. (2004) compared CHAMP dry temperature with interpolated data from ECMWF meteorological analyses with co-located and near simultaneous radiosonde observations between 10 and $35 \mathrm{~km}$. Biases between ECMWF and CHAMP RO profiles are less than $0.5 \mathrm{~K}$, while comparison using radiosonde observations and RO profiles indicates nearly no bias between 
approximately 9 and $27 \mathrm{~km}$. This lack of bias between the observational data sets suggests deviations of the analyses from the real atmospheric situation. Work by Gobiet et al. (2005) which compares ECMWF operational analyses with CHAMP RO data found close overall agreement worldwide, apart from discrepancies in the Antarctic stratosphere where cold biases up to $-2.5 \mathrm{~K}$ and warm biases up to $+3.5 \mathrm{~K}$ were observed.

These and other previous analyses led Wang and Lin (2007) to use COSMIC RO soundings to examine the distribution of the coldest atmospheric temperatures in the Antarctic stratosphere. Their study suggests that these high quality measurements of vertical temperature structure over the Antarctic region are potentially important inputs for models that predict polar stratospheric clouds occurrence and stratospheric ozone depletion (Huck et al., 2005). Very recent work by Juarez et al. (2009) indicated that CHAMP $\mathrm{RO}$ observations are able to detect more PSC-prone temperature profiles during winters with disturbed conditions in the Arctic than the ECMWF analyses. Those authors consider that this is due to the ability of CHAMP RO to detect shortvertical wavelength features which may represent either localized gravity or global-scale planetary waves.

In this study, CHAMP observations are utilized to quantify the magnitude of the background temperature and perturbations associated with gravity waves. However, the resolution of the CHAMP observations has a direct impact on the observability of different portions of the gravity wave spectrum and this needs to be considered. Work by Lange and Jacobi (2003) shows that while the weighting function of the RO measurements is $200-400 \mathrm{~km}$ along the LOS, the GPS measurements are sensitive to gravity waves with horizontal wavelengths greater than $100 \mathrm{~km}$ and vertical wavelengths between approximately 1.4 and $10 \mathrm{~km}$. This is partially associated with the LOS having a parabolic path in the atmosphere and also the fact that the plane waves visible will depend on the angle between the LOS and the wavefronts. When the LOS is perpendicular to the wavefronts then spatial averaging will significantly affect the wave activity observed. In particular, work by de la Torre and Alexander (2005) has shown that the observability of mountain waves, which have horizontal wavelengths of tens to hundreds of kilometers, can be significantly impacted. Further work by Baumgaertner and McDonald (2007) suggested that the preponderance of LOS within 30 degrees of the north/south axis in the Antarctic region means that the viewing geometry over the Antarctic Peninsula and the Trans-Antarctic Mountains potentially favoured the detection of the mountain waves by CHAMP in these regions. However, all this previous work suggests that CHAMP RO measurements observe a limited portion of the gravity wave field. A study detailed in McDonald and Hertzog (2008) which compared coincident CHAMP and in-situ super-pressure balloon measurements of the gravity wave field suggests that overall the wave field is such that CHAMP underestimates the magnitude of the potential en-

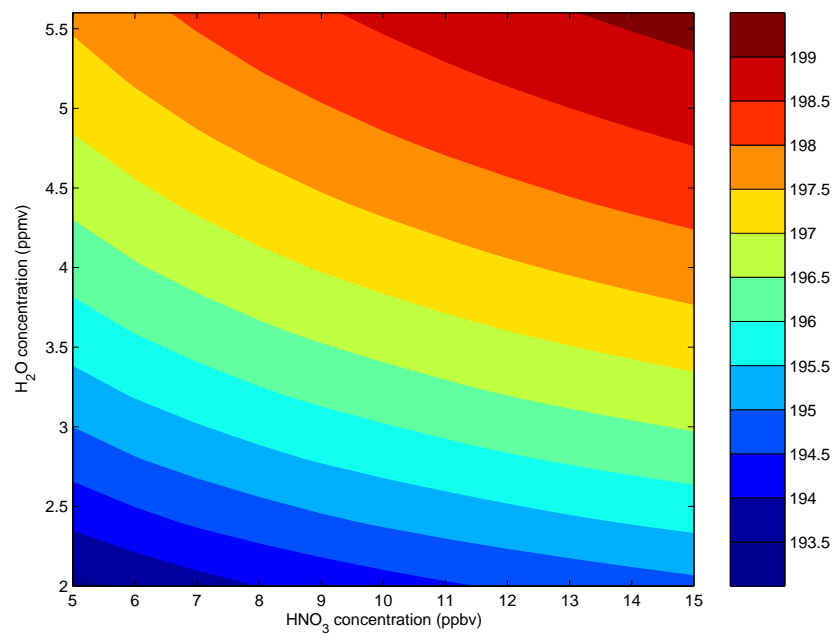

Fig. 2. Contour plot of the NAT PSC formation temperature (Kelvin) as a function of nitric acid and water mixing ratios at $18 \mathrm{~km}$ (Function based on Hanson and Mauersberger (1988)).

ergy per unit mass. Thus, in any analysis CHAMP would likely provide a conservative estimate of the impact of temperature perturbations associated with gravity waves. Ancillary profiles of temperature and pressure from the Met Office analyses which have been interpolated to the location and time of the POAM III measurements have also been used in this study. The details of the Met Office analyses are described in Lorenc et al. (2000).

\subsection{PSC temperature thresholds}

In our analysis temperatures obtained from CHAMP observations and Met Office analyses are used to determine the frequency of temperatures below various temperature thresholds. The NAT formation temperature, $T_{\mathrm{NAT}}$, at $18 \mathrm{~km}$ as derived using the formulation of Hanson and Mauersberger (1988) which is a function of nitric acid, water vapour concentrations and pressure is shown in Fig. 2, the ranges selected are typical of concentrations in the polar stratosphere. Figure 2 clearly shows that $T_{\text {NAT }}$ varies much more as a function of water vapour than nitric acid mixing ratios for the ranges typical of the Antarctic stratosphere chosen in this diagram. The selection of these values is based on nitric acid concentrations identified in Santee et al. (2007) and typical water vapour concentrations at this altitude measured by the POAM III instrument (see Fig. 3). The probability of crossing the NAT formation temperature threshold is thus determined by comparing Met Office and CHAMP temperature observations at a specific altitude with the $T_{\mathrm{NAT}}$ threshold derived using the formulation of Hanson and Mauersberger (1988) for that altitude. The $\mathrm{H}_{2} \mathrm{O}$ variation used is taken from the POAM III observations and a constant nitric acid mixing ratio of $10 \mathrm{ppbv}$ is assumed unless indicated otherwise in the text. As previously indicated this study assumes the 


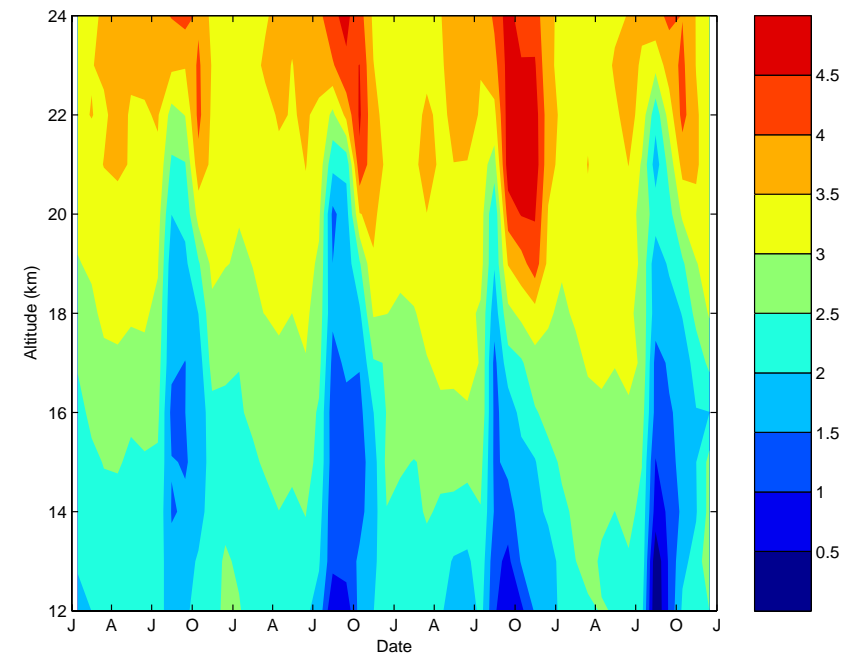

Fig. 3. Time-altitude contour plot of the $\mathrm{H}_{2} \mathrm{O}$ mixing ratio (ppmv) derived from POAM III observations for the period January 2002 to December 2005.

STS formation temperature occurs at $3.5 \mathrm{~K}$ below the value of $T_{\text {NAT }}$ (Carslaw et al., 1994) and the ice frost point temperature is derived using the empirical formulae identified in Marti and Mauersberger (1993).

A time-altitude contour plot of the water vapour mixing ratio for the period January 2002 to December 2005, used in the temperature threshold calculations utilized in the rest of this paper, is shown in Fig. 3. Examination of the seasonal variation of water vapour mixing ratio displays a consistent irreversible dehydration potentially associated with the sedimentation of ice PSC at altitudes between 12 and $20 \mathrm{~km}$ between August and September each year. Nedoluha et al. (2003) indicates that the higher water vapour mixing ratios observed at elevated altitudes are likely to be associated with moist air moving into the region via diabatic descent.

Uncertainties on the probabilities displayed later in this study are derived assuming a binomial distribution in a similar manner to that utilized in Alfred et al. (2007). The standard deviation of a binomial distribution, $\sigma$, can be written as:

$\sigma=\sqrt{N p(1-p)}$

where $N$ is the number of observations and $p$ is the probability. This number indicates the number of observations that might occur by chance and thus needs to be divided by the total number of observations to determine the uncertainty on the probability.

\section{Results}

Figure 4 displays a time-altitude contour plot of the median Met Office analysis temperature interpolated to the positions

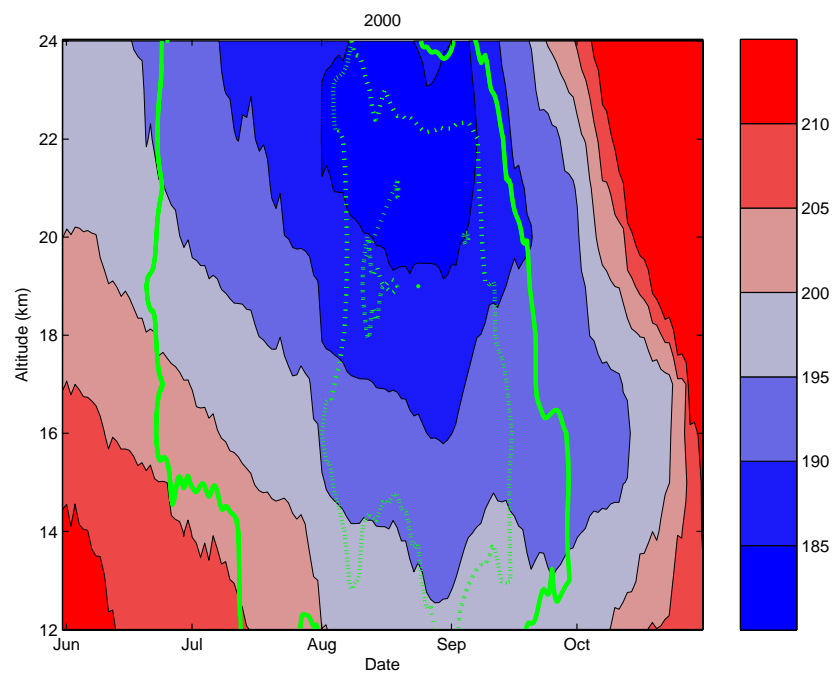

Fig. 4. Time-altitude contour plot of the median temperature (Kelvin) derived from Met Office analyses data interpolated to POAM III measurement locations in 2000. The full green line indicates the $25 \%$ PSC probability contour and the dotted green line indicates the $75 \%$ PSC probability contour, these contours being derived from POAM III extinction measurements. Note that the tick marks on the date axis indicate the first day of the month.

of POAM III observations for 2000. Overlaid are the 25 and $75 \%$ PSC probability contours determined from POAM III extinction measurements. To ensure a large enough statistical sample, probabilities are calculated using a sliding 10-day window. Examination of Fig. 4 suggests a qualitative correspondence between cool temperatures and PSC occurrence.

Figure 5 displays the probability of temperatures below $T_{\text {NAT }}$ based on Met Office analyses (red line), the STS PSC formation temperature (green line), $T_{\mathrm{STS}}$, and the ice nucleation temperature (blue line), $T_{\mathrm{ICE}}$, against time of year. Figure 5 also displays the probability of PSC detection based on the k-means detection algorithm applied to POAM III observations (black line). The latter also includes additional regions identified using the ZMIN quality flag (reducing the underestimation of Type II PSC). It should be noted that the values in Fig. 5 are the probabilities of PSC occurrence at $18 \mathrm{~km}$ derived over a 10 day interval. This methodology should provide accurate values of both PSC occurrence and temperature threshold crossings, but the daily values plotted will not be independent. Statistical uncertainties are calculated as a three-standard-deviation envelope around each of the average series (displayed, for reference purposes, on the fifteenth day of each month). Note that problems with the POAM III instrument and quality control procedures produce data gaps in several years observations, most noticeably 2004 (see Fig. 5). Examination of Fig. 5 shows the expected increase in PSC incidence from June to September and a general reduction in occurrence after this date associated with changes in temperature, nitric acid and water 

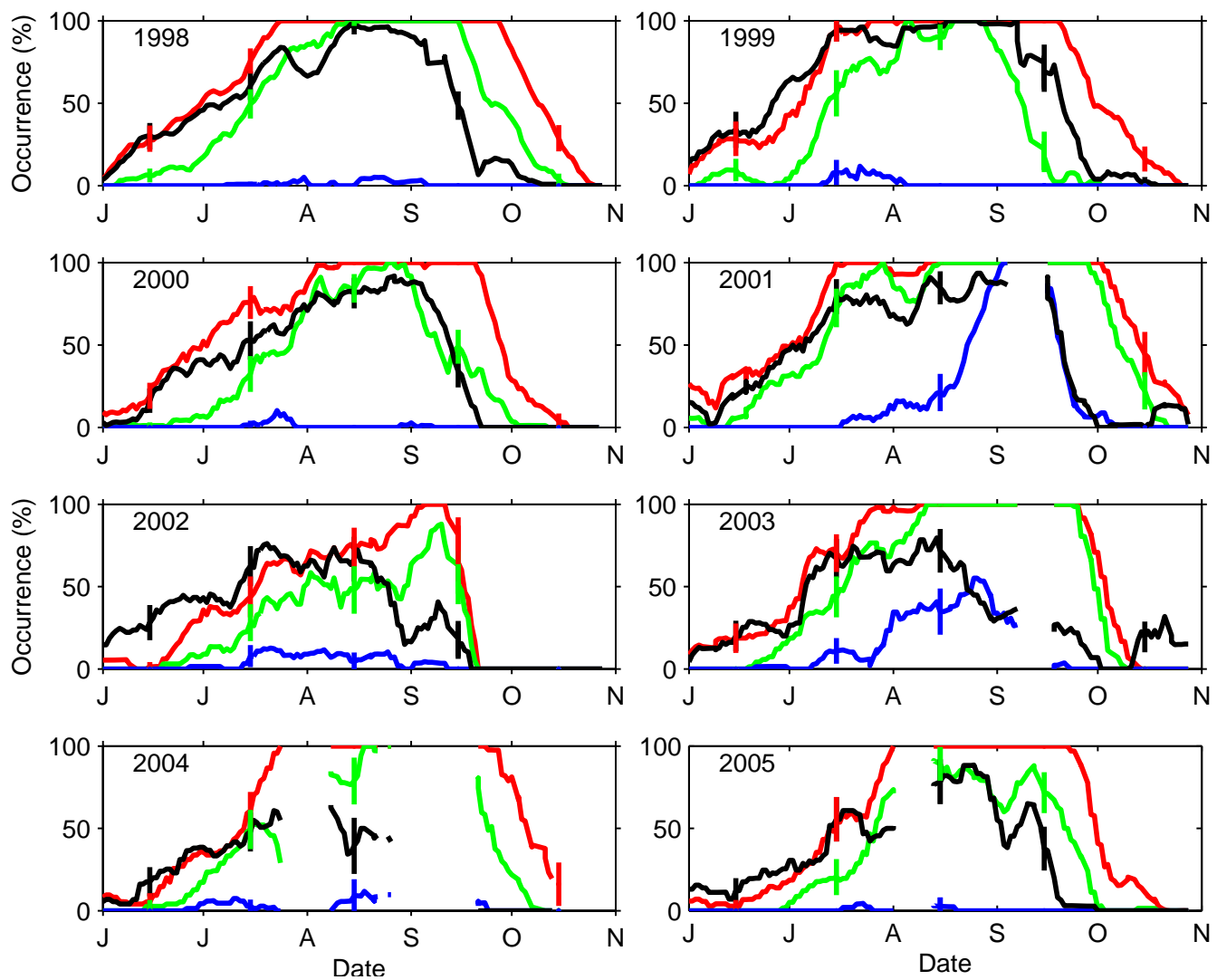

Fig. 5. The probability of temperatures below the $T_{\mathrm{NAT}}$ threshold derived from Met Office analysis and using the $\mathrm{H}_{2} \mathrm{O}$ variation derived from the POAM III observations and a constant nitric acid mixing ratio of $10 \mathrm{ppbv}$ (red line), the probability of temperatures below the $T_{\mathrm{STS}}$ threshold (green line),the probability of temperatures below the $T_{\mathrm{ICE}}$ threshold (blue line) and the probability of PSC derived from POAM III extinction measurements (black line) against time of year for the 8 years of POAM III observations. It should be noted that this is the probability at $18 \mathrm{~km}$ derived over a sliding time window of 10 days. The vertical lines on the fifteenth of each month display the plus and minus three standard deviations for each probability. Note that the tick marks on the date axis indicate the first day of the month.

vapour concentrations. However, comparison of the PSC occurrence predicted from temperature threshold crossings and those derived from POAM III extinction data do not correspond within the defined uncertainties in several regions.

Averaged over the entire period identified the $T_{\mathrm{NAT}}$ temperature threshold derived from Met Office analyses predicts more PSC than observed by POAM III. This is to be expected because Type Ia PSC formation requires average temperatures below the $T_{\mathrm{NAT}}$ threshold for a period. The exact temperature value required varies dependent on whether NAT only forms by heterogeneous nucleation on pre-existing ice particles, requiring temperatures below $T_{\mathrm{ICE}}$ (Carslaw et al., 1994), or NAT nucleation mechanisms independent of the existence of ice particles (Hitchman et al., 2003; Pagan et al., 2004; Svendsen et al., 2005). The POAM III observations predict between 60 and $85 \%$ of the occurrence identified by the $T_{\text {NAT }}$ temperature threshold in all the years except 2004 where only $45 \%$ of the PSC predicted by the $T_{\mathrm{NAT}}$ threshold is observed. Comparison of the PSC occurrence observed by POAM III and identified by the $T_{\mathrm{STS}}$ temperature threshold displays a better correspondence, but this threshold still overestimates the quantity of PSC. It should be noted that this result concurs with recent CALIPSO observations detailed in Pitts et al. (2007) which suggest that the $T_{\mathrm{STS}}$ temperature threshold may be a better measure of PSC existence than the $T_{\text {NAT }}$ threshold. Inspection of the frequency of PSC occurrence identified by the $T_{\mathrm{ICE}}$ threshold derived from analyses and the POAM III frequency suggests the $T_{\mathrm{ICE}}$ occurrence generally significantly underestimates the quantity of PSC observed by POAM III.

Close examination of Fig. 5 indicates that, during certain years (specifically 1999 and 2002 to 2005 inclusive), the observed quantity of PSC in June is greater than that predicted from $T_{\mathrm{NAT}}$ and $T_{\mathrm{STS}}$ temperature thresholds alone. Analysis (not shown here) indicates that this pattern does not change significantly even if the nitric acid mixing ratio is increased to $15 \mathrm{ppbv}$; this change would make the temperature threshold warmer and thus easier to cross. It is also noticeable that at all other periods of the year the probability of PSC occurrence from POAM III measurements is smaller than the 
expected value based on the proportion of observations below the $T_{\mathrm{NAT}}$ threshold. The probability of PSC occurrence based on the $T_{\text {ICE }}$ temperature threshold does not exceed the probability of occurrence based on POAM III observations in any of the eight observational years until after mid-August. Comparison of PSC occurrence based on POAM III observations with the quantity of Met Office analysis data below the $T_{\mathrm{STS}}$ threshold shows a greater, but not perfect, correspondence which is generally best between the start of July and the start of September. It should be noted that the temperature thresholds determined account for variations in the water vapour mixing ratio based on POAM III observations, but that the nitric acid is held constant at 10 ppbv. Previous studies suggest that the difference between the occurrence probability based on POAM III observations and those based on temperature thresholds later in the season is due to denitrification and dehydration (Nedoluha et al., 2003; Alfred et al., 2007). Thus, the fixed nitric acid concentrations used in Fig. 5 are likely to explain some of the over-estimation observed after June. Note also that while not shown here Type Ia PSCs are identified almost exclusively by the unsupervised clustering algorithm in June and early July in all years.

As previously indicated NAT particles probably normally form at temperatures several degrees colder than $T_{\mathrm{NAT}}$ (Tabazadeh et al., 2001) or at even lower temperatures (Carslaw et al., 1998). Therefore, a high probability of PSC formation would not be expected in June even if the probability of temperatures crossing the $T_{\mathrm{NAT}}$ temperature threshold was relatively large unless a mechanism for Type Ia cloud formation that requires temperatures only below $T_{\mathrm{NAT}}$ exists. This study examines whether the enhanced PSC occurrence observed can be explained by gravity wave motions. It should be noted that previous work by Saitoh et al. (2006) has identified a similar enhanced PSC occurrence in June 2003 compared to that identified by temperature thresholds using ILAS-II data. An enhanced level of PSC occurrence in June 2003 was also observed and modelled by Höpfner et al. (2006). Their study showed that only heterogenous nucleation on ice formed in mountain waves could reproduce the observed PSC morphology. This analysis has also recently been confirmed by AIRS observations discussed by Eckermann et al. (2009).

Poole and Pitts (1994) have examined the relationship between PSC frequency and temperature previously and shown that PSC formation in the Southern Hemisphere is less likely later in the PSC season than earlier. Other studies, such as Mergenthaler et al. (1997) have suggested that PSC formation late in the PSC season in the Southern Hemisphere is strongly affected by the preceding denitrification and dehydration which fits with the observations displayed in Fig. 3 and 5. Saitoh et al. (2006) also suggests that PSC frequency depends on the degree of denitrification later in the season. Their study also indicates that $T_{\mathrm{NAT}}$ is not a good measure of PSC occurrence especially above $20 \mathrm{~km}$ in late winter.
A potential explanation for the differences between the PSC occurrence rates derived from temperature thresholds using Met Office analyses and those derived from POAM III extinction data could be seasonally varying biases in the Met Office observations. For example, a warm bias in the Met Office temperatures in June would produce lower PSC occurrence rates than the true values which might explain the discrepancies observed in some years in this period. Figure 6 therefore compares Met Office analyses and CHAMP RO temperatures at the POAM III sampling latitudes (see Fig. 1). It should be noted that studies by Parrondo et al. (2007) and Luntama et al. (2008) have previously shown that CHAMP observations provide good estimates of the background temperatures which can be used to identify issues in reanalyses datasets. Figure 6 displays time-altitude contour plots of the median temperature observed by Met Office analyses and CHAMP RO observations in 2005. The black contour line in both panels of Fig. 6 displays the $195 \mathrm{~K}$ contour line from the Met Office analyses for reference purposes. In this case, Figure 6 is used to identify whether the increased $T_{\text {NAT }}$ occurrence probability observed in June may be associated with biases between the temperatures identified in the Met Office analyses and the CHAMP RO observations. We remove the effect of gravity wave perturbations on the CHAMP RO observations by using a 4th order polynomial filter. Examination shows a good correspondence between CHAMP RO and Met Office analyses in June, though detailed examination suggests the CHAMP RO observations are slightly cooler. In July to August the temperatures differences are negligible and in September and October the temperatures of the Met Office analyses are slightly cooler than the CHAMP observations. Over the entire period the mean bias between the two sets of data is very small $(\sim-0.08 \mathrm{~K})$. Similar analysis for 2002, 2003 and 2004 shows mean differences of -0.24 , 0.2 and $0.47 \mathrm{~K}$, respectively. The similarity of the bias in each year compared to the change in the difference between the estimated PSC occurrence based on temperature information and POAM III observations suggests that the difference can not be solely explained by bias in the Met Office temperatures.

This study now examines the potential of gravity waves to cause more temperature threshold crossings than expected from the mean represented by Met Office analyses and in particular attempts to identify whether the enhanced PSC frequency in June may be associated with temperature variations that are not resolved in the Met Office analyses. To examine this we use high vertical resolution measurements made by the CHAMP RO instrument available between 2002 and 2005. It should be noted that the CHAMP observations are not dense enough to directly identify the presence of gravity waves at POAM III measurement locations and thus we are limited to examining temperature threshold crossing frequencies. 

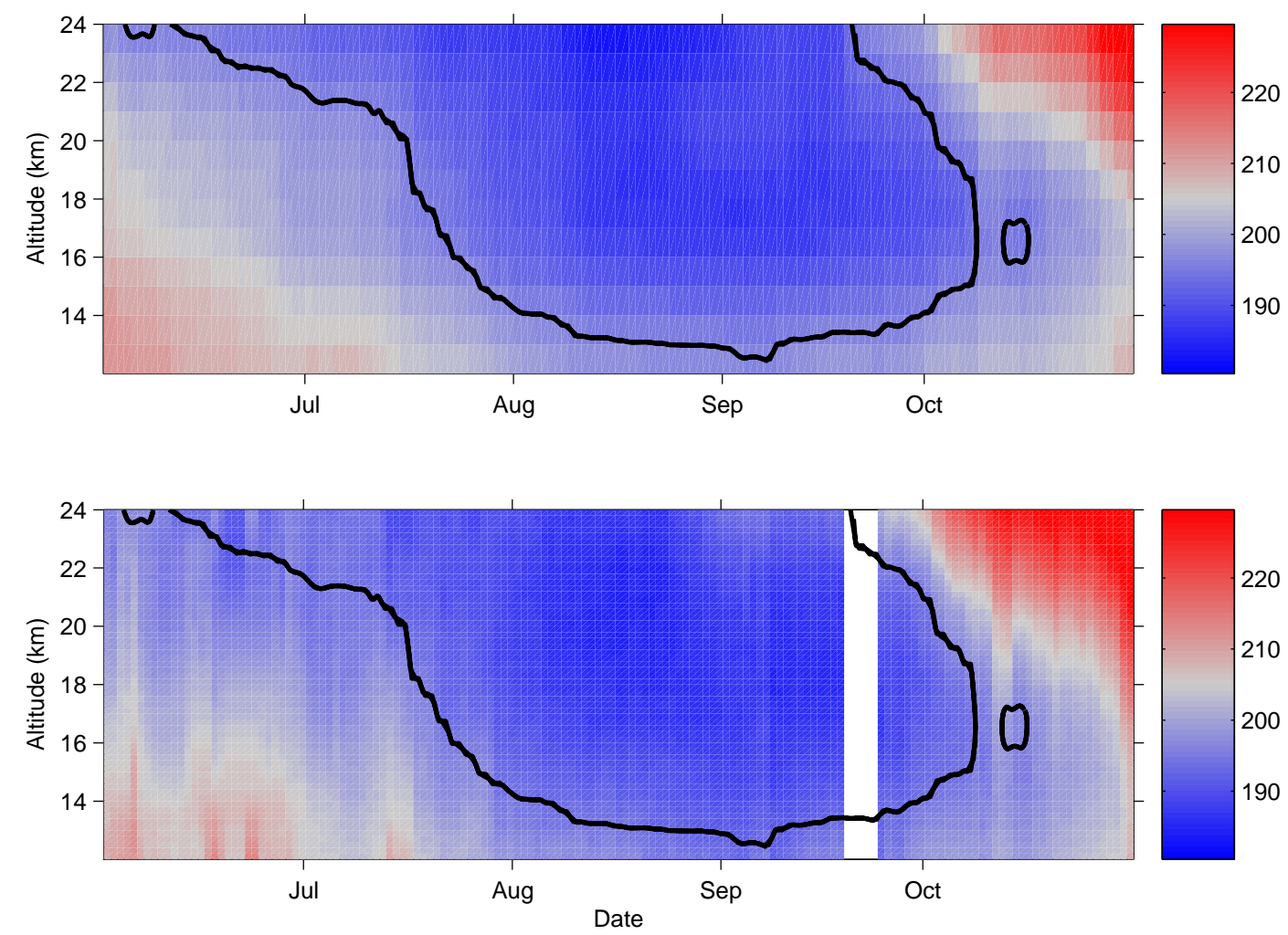

Fig. 6. Time-altitude contour plot of the median temperature (Kelvin) derived from Met Office analysis (top panel) and CHAMP radio occultation observations (bottom panel). The black contour line in both panels displays the 195K contour line from the Met Office analysis. Note that the tick marks on the date axis indicate the first day of the month.

We begin by displaying a single CHAMP temperature profile observed on 2 June 2006 (see Fig. 7). Figure 7 also displays the mean temperature derived by applying a 4th order polynomial fit to the CHAMP observations (red line) and the $T_{\mathrm{NAT}}$ (blue line) and $T_{\mathrm{ICE}}$ (blue dashed line) temperature thresholds. This temperature profile displays a clear wavelike structure. The temperature perturbations associated with the gravity wave (the difference between the red and green lines) allow the $T_{\mathrm{NAT}}$ threshold to be crossed at around $17 \mathrm{~km}$ and between approximately 20 and $21 \mathrm{~km}$, while the mean temperature profile does not cross the $T_{\mathrm{NAT}}$ threshold. It should also be noted that the temperature perturbations, typically $\pm 2 \mathrm{~K}$, do not cross the $T_{\text {ICE }}$ threshold. This is typical in CHAMP observations for this period of the year, but periods where the temperature perturbations associated with gravity waves allow a crossing of the $T_{\mathrm{STS}}$ or the $T_{\mathrm{ICE}}$ threshold do occur. It should be noted that the magnitude of the temperature perturbations observed corresponds well to those identified in Innis and Klekociuk (2006). However, the impact of observational filtering on CHAMP measurements will mean that these observations under-represent the true magnitude of temperature perturbations associated with portions of the gravity wave spectrum. Thus, it is not possible to guarantee that the true minimum temperatures are not significantly cooler than those observed. The analysis in Lange and Jacobi (2003) suggests that CHAMP observations are likely to provide a lower limit on the impact of gravity waves on PSC formation. It should also be noted that while not perfect the relatively high resolution of CHAMP data in the vertical and horizontal resolution compared to model resolution does allow fine-scale structure not observed in analyses to be examined (Juarez et al., 2009).

Figure 8 displays temperature distributions relative to $T_{\text {NAT }}$ taken from CHAMP RO observations at $18 \mathrm{~km}$ for different months and latitudinal bands. The aim of Fig. 8 is to show the temporal and latitudinal structure of temperatures conducive to PSC formation unhindered by the POAM sampling pattern and to be of use for comparisons with Fig. 5. Given the relatively small number of CHAMP profiles in each month observed poleward of $60^{\circ} \mathrm{S}$, generally less than 700 , we have used coarse ten degree wide latitudinal bands to ensure significant numbers of points in each histogram. The use of these wide regions make it more difficult to compare directly with the Met Office analyses, but given the limited intra-monthly CHAMP data and the more detailed analysis discussed later we believe this is not a significant issue. This coarse latitudinal sampling also has the benefit of reducing any affects associated with a distorted vortex. Examination 


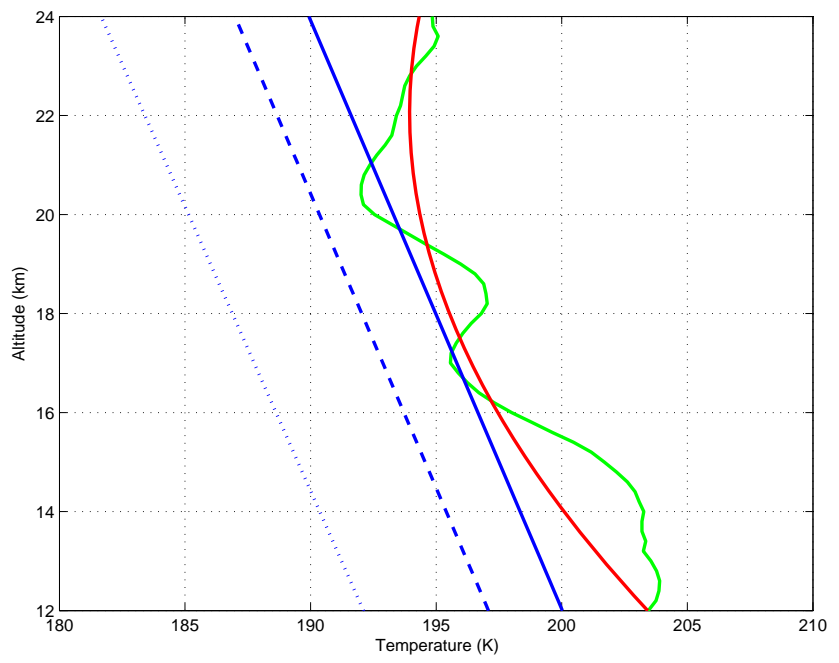

Fig. 7. This diagram shows a CHAMP temperature profile from 2nd June 2006 (green line) and a 4th order polynomial fit to the CHAMP data (red line). The polynomial fit approximates the mean temperature observed by CHAMP, the difference between the red and green lines represents temperature perturbations due to gravity waves. The blue full, dashed and dotted lines indicate the values of the $T_{\mathrm{NAT}}, T_{\mathrm{STS}}$ and $T_{\mathrm{ICE}}$ temperature thresholds, respectively.

of Fig. 8 shows a clear latitudinal variation with colder temperatures observed towards the pole. A seasonal variation with coldest temperatures observed in July and August is also observable in each latitude band. Examination of the temperature relative to the $T_{\mathrm{NAT}}$ temperature threshold shows that in July and August at latitudes poleward of $70^{\circ} \mathrm{S}$ nearly all the temperatures observed are below $T_{\mathrm{NAT}}$. Given that temperature perturbations associated with gravity waves are most likely to have a significant impact on PSC occurrence when the climatological mean is close to a PSC temperature threshold it is important to identify the dates and latitudes when these conditions occur. These conditions preferentially occur between $60^{\circ}$ to $70^{\circ} \mathrm{S}$ in June through August, in the $70^{\circ}$ to $80^{\circ} \mathrm{S}$ range in August to September and in the $80^{\circ}$ to $90^{\circ}$ range in October. Comparison of this variation with the POAM latitudinal sampling pattern displayed in Fig. 1 shows a quite good agreement until the end of September. Thus, while the POAM observations are clearly not representative of the conditions associated with the whole vortex, as previously identified by Pitts et al. (2007) for solar occultation sampling, they actually follow the region where temperatures may be conducive for gravity wave induced PSC formation quite well at this altitude. However, it should be noted that this correspondence is not perfect and is only relevant to the $T_{\text {NAT }}$ threshold and the POAM sampling does not observe the Antarctic peninsula in August and September when there is still a possibility of significant gravity wave impacts. The general consistency of these patterns from year to year is also clear, with the exception of 2002, on examination of Fig. 8.
A simple comparison of the occurrence of temperatures below $T_{\text {NAT }}$ shows that in June 2002 CHAMP RO observations occur $29 \%$ of the time, Met Office analyses have a mean occurrence of approximately $20 \%$ over the month and the POAM III extinction data suggests an occurrence rate of roughly $30 \%$. A closer correspondence between the CHAMP RO and POAM observations is therefore apparent. In 2003 CHAMP RO observations occur $30 \%$ of the time, Met Office analyses have a mean occurrence of approximately $20 \%$ over the month and the POAM extinction data have an occurrence rate of roughly $20 \%$. In this case, the CHAMP RO observations suggest a higher PSC occurrence than the POAM observations. In 2004 CHAMP RO observations suggest temperatures below $T_{\text {NAT }}$ occur $23 \%$ of the time, Met Office temperatures are below the NAT threshold about $15 \%$ of the time and POAM III data identifies PSC 19\% of the time. Similar results occur in 2005 with a slightly closer correspondence between the CHAMP RO observations and the POAM III values. This simple analysis indicates that in some cases CHAMP RO has the potential to provide better estimates of temperature than those of the Met Office analysis. It is interesting that in other months and latitudinal bands the probabilities determined from CHAMP RO data are larger than those derived from POAM III observations. Given the work detailed in Juarez et al. (2009) the slightly better relationship in each June could be associated with the ability of CHAMP RO measurements to observe fine-scale structure not included in analyses. The enhanced PSC occurrence in the $60^{\circ}$ to $70^{\circ}$ latitude range in June may be evidence that the gravity wave "hotspot" of the Antarctic peninsula (Baumgaertner and McDonald, 2007; Hertzog et al., 2008) could be particularly important in this period. Basically, the combination of mean temperatures close to the $T_{\mathrm{NAT}}$ threshold and large mountain wave temperature perturbations could be important. The over-estimation of PSC by CHAMP RO observations in some years in June, notably 2003, could be associated with a number of possible factors. The over-estimation could be associated with the fact that temperatures below $T_{\text {NAT }}$ do not necessarily lead to NAT formation straight away (Peter, 1997). The over-estimation could also be associated with differences in the spatial and temporal distribution of CHAMP and POAM III observations. Another possibility is that the limited longitudinal sampling of POAM III observations may underestimate PSC in some years. It should be noted that the consistent over-estimation of PSC frequency by CHAMP RO compared to that identified using POAM III is perhaps expected. More consistent over-estimation of the true PSC occurrence based on temperature thresholds makes physical sense given that these thresholds are a necessary but not sufficient condition for PSC formation. 

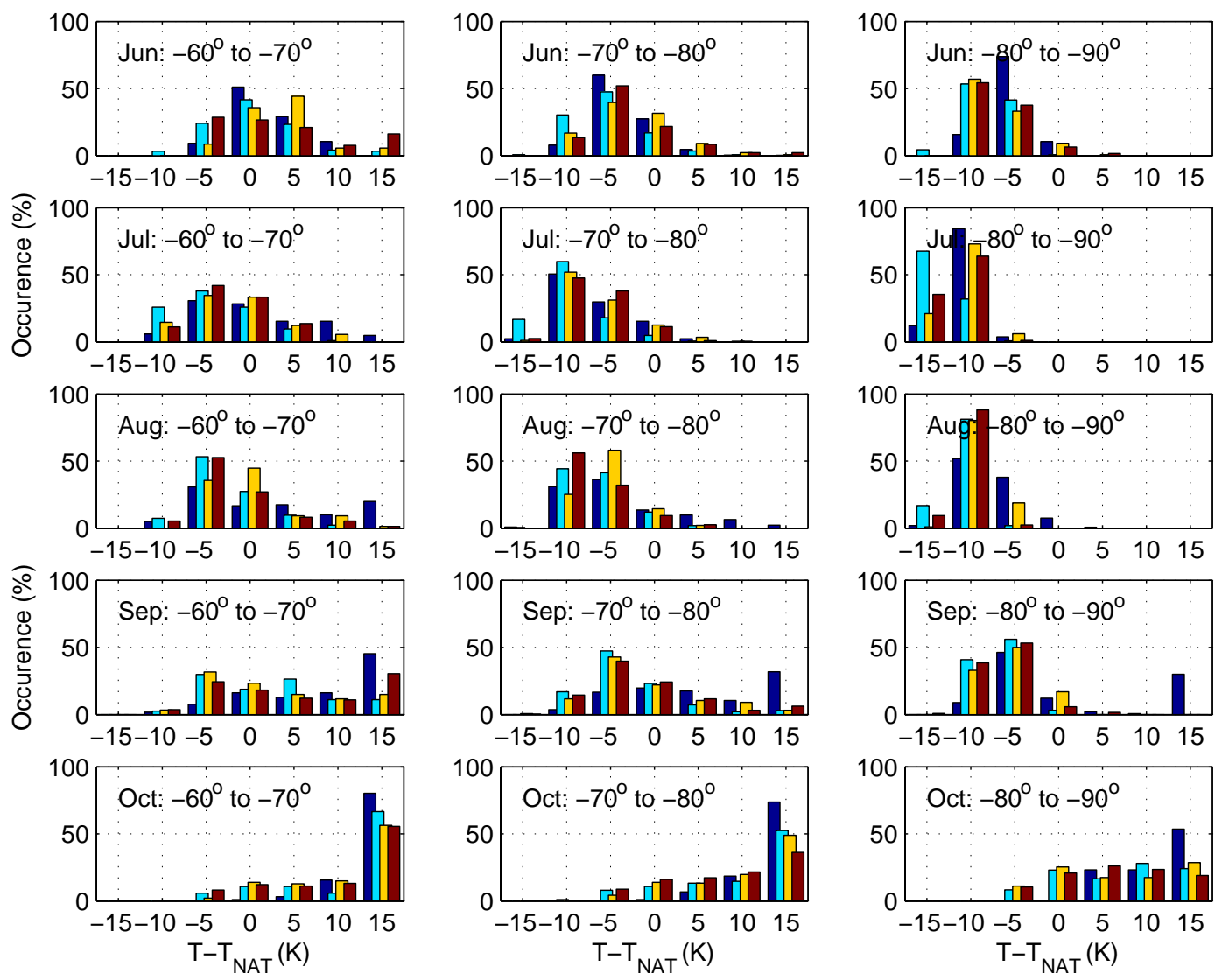

Fig. 8. The set of diagrams displayed show the temperature distribution relative to $T_{\mathrm{NAT}}$ observed at $18 \mathrm{~km}$ by CHAMP RO observations for different months and latitudinal regions (indicated on each diagram). The different color bars represent data from different years, these being 2002 (dark blue), 2003 (light blue), 2004 (yellow) and 2005 (red).

\section{Discussion}

As previously indicated temperature biases may explain some of the differences observed between temperature threshold crossing frequencies and the frequency of PSC observed by POAM III extinction measurements. However, further inspection suggests that some features of the data may point to a role for internal gravity wave temperature perturbations. Figure 9 displays a time-longitude contour plot for 2005 of the probability of PSC formation based on POAM III observations. Examination of Fig. 9 shows that an enhanced PSC probability, which relates to the enhancements in June described previously in relation to Fig. 5, occurs around a longitude of $300^{\circ} \mathrm{E}$. This longitude corresponds to the position of the Antarctic peninsula which has previously been identified as a "hotspot" for gravity wave activity (Wu and Jiang, 2002; Baumgaertner and McDonald, 2007; Hertzog et al., 2008) and therefore might be a potential region of enhanced PSC occurrence. A distinct wavenumber one signature is observable later in the season which is likely to be caused by planetary waves.
Thus, the expectation that a region of high gravity wave activity might be associated with 'enhanced' PSC occurrence seems to be confirmed. It should be noted that MIPAS measurements have identified enhanced PSC occurrence associated with mountain waves in this area earlier in the same month in 2003 (Höpfner et al., 2006). This suggests that mountain waves may play a significant role in enhanced PSC formation and that this enhancement may occur frequently in this region and period. This is supported by the fact that the time-longitude contour plot for 2003 derived from POAM III data (not shown) displays a similar pattern to that observed in Fig. 9. However, it should be noted that the background temperatures shown in Fig. 8 display a large proportion of observations close to the NAT formation temperature ( $\left.T_{\mathrm{NAT}}\right)$ in this period. Thus, the observed enhancement is likely to be a result of both these factors.

As previously indicated Figure 5 shows that during June the number of PSC observed is greater than expected based on the occurrence of regions where the $T_{\mathrm{NAT}}$ and $T_{\mathrm{STS}}$ temperature thresholds are crossed in some years, most clearly 2002 and 2005 but also for a short period in 2003. A previous study by Saitoh et al. (2006) using ILAS-II observations from 


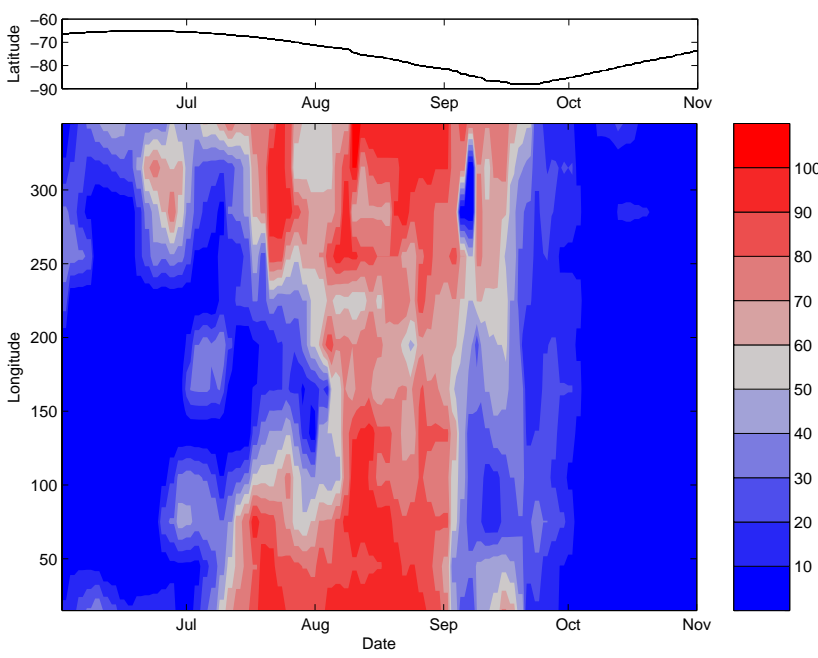

Fig. 9. A plot of the latitude of observations as a function of date (upper panel) and a date-longitude contour plot of the percentage probability of PSC observation derived from POAM III measurements (lower panel) for the year 2005. Note that the tick marks on the date axis indicate the first day of the month.

2003 shows a corresponding enhanced PSC frequency compared to that expected based on temperature thresholds. This is a strong validation of the results presented in this study since Saitoh et al. (2006) uses the mean plus five standard deviations from the ILAS-II aerosol extinction data to identify PSC, an extremely conservative threshold.

To examine the importance of gravity wave temperature perturbations on temperature threshold crossings we can use CHAMP RO observations with and without these temperature perturbations included. Figure 10 displays the probability of temperatures below the $T_{\mathrm{NAT}}$ temperature threshold against the probability of the threshold crossing being associated with a gravity wave perturbation based on CHAMP RO observations for 2002 to 2005 . The second parameter was calculated by identifying all those temperature profiles where the $T_{\mathrm{NAT}}$ threshold was crossed and comparing whether the $T_{\mathrm{NAT}}$ threshold was crossed if only the mean temperature structure, identified using a 4th order polynomial fit to the CHAMP profile and thereby removing small vertical scale motions, was observed. By comparing the number of threshold crossings associated with the smoothed profile and the unfiltered profile we can determine the proportion of $T_{\mathrm{NAT}}$ crossings which occur because of temperature perturbations. Figure 10 suggests that temperature perturbations associated with gravity waves account for approximately $40 \%$ of $T_{\mathrm{NAT}}$ temperature threshold crossings in every June observed (2002 to 2005) and roughly 15\% in all other months and years. It should be noted that only CHAMP profiles in the same latitude region that POAM III observations examine have been used in this analysis. Figure 10 suggests that gravity waves may have a significant impact early in the

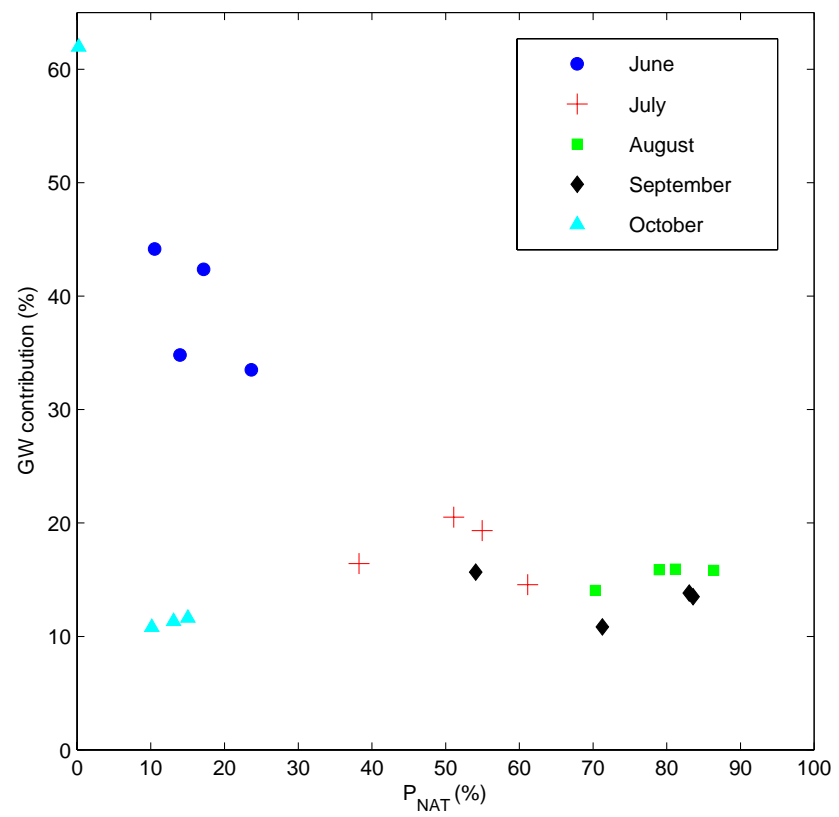

Fig. 10. Probability of observations of temperatures below the NAT nucleation temperature $T_{\mathrm{NAT}}$ from CHAMP against the contribution from small-scale wave motions (gravity waves) for different months indicated in the legend. It should be noted that the individual points for a particular month relate to data from different years.

PSC formation season when the mean temperature is close to the $T_{\mathrm{NAT}}$ threshold. This may explain the very clear identification of mountain waves as the source of PSC detailed in Höpfner et al. (2006). The value for the months other than June compare extremely well with the estimate indicated in Innis and Klekociuk (2006) that gravity wave perturbations influence PSC formation approximately $15 \%$ of the time.

Examination of the quantity of data close to the $T_{\mathrm{NAT}}$ threshold in Fig. 8 corroborates the estimates of the gravity wave influence derived in Fig. 10. In particular, when POAM observes between $65^{\circ}$ and $70^{\circ} \mathrm{S}$ the quantity is close to $40 \%$ in June and between 10-20\% for July to October when examined at the POAM sampling latitudes. Another interesting point identified by a reviewer of this paper is that we can then infer from Fig. 8 that the gravity wave impact on NAT nucleation should be around 20\% for August and September above the Peninsula.

Similar analysis to that shown in Fig. 10 for the $T_{\mathrm{STS}}$ and $T_{\text {ICE }}$ temperature thresholds shows comparable results; that is an increased frequency of crossing the thresholds associated with gravity wave perturbations. However, the seasonal patterns are shifted towards colder months as might be expected. In addition, no extra threshold crossings associated with $T_{\mathrm{STS}}$ and $T_{\mathrm{ICE}}$ are observed in June. Given that NAT particles are usually considered to form at temperatures several degrees colder than $T_{\mathrm{NAT}}$ (Tabazadeh et al., 2001) or at even lower temperatures (Carslaw et al., 1998) the quantity 
of PSC observed by POAM III is a little surprising and possibly supports studies such as Voigt et al. (2005) which suggest that there is a mechanism for NAT PSC formation that requires temperatures no lower than a few degrees below $T_{\mathrm{NAT}}$. But, this result is dependent on the level of observational filtering in CHAMP measurements and could also be impacted by the constant value of nitric acid assumed in this work.

As previously indicated observational filtering means that CHAMP RO measurements observe only a portion of the actual gravity wave spectrum (Lange and Jacobi, 2003). The finite spatial resolution of the CHAMP observations means that any temperature perturbation observed can be thought of as a spatial average of the temperature perturbations over the satellite's weighting function. This spatial averaging thereby reduces the variance observed. The study by Lange and Jacobi (2003) using a simple assumed gravity wave field, which has a gravity wave spectrum with constant amplitude in the range $100-1000 \mathrm{~km}$ horizontal and $1-10 \mathrm{~km}$ vertical wavelength, indicates that about $80 \%$ of the total variance is retrieved in the worst case when the LOS scans perpendicular to the wave crests and about $88 \%$ when the LOS through the gravity waves is arbitrarily oriented. Assuming a more realistic vertical wavelength spectrum with a spectral gradient of $-5 / 3$ the mean observed variance reduces by an additional $2-3 \%$. However, PSC formation will be impacted only by regions where the temperature is sufficiently cool for formation to occur. Thus, since the POAM III instrument is also a limb sounder (and effectively produces a spatially averaged extinction) it will observe the integrated affect of a heterogeneous temperature field on PSC occurrence. Given high resolution lidar observations of heterogeneous structures associated with PSC fields display clear phase structures related to gravity waves (Shibata et al., 2003). We would suggest that the spatial average temperature field may be a useful measure of the PSC formation potential averaged over an area. However, it should be noted that this measure is by no means perfect because the nonlinear hysteresis affect inherent in PSC microphysics would impact the spatial average.

\section{Conclusions}

Examination of Fig. 5 shows that PSC occurrence can be relatively well predicted by the presence of temperatures below either the $T_{\mathrm{NAT}}$ or $T_{\mathrm{STS}}$ thresholds. This is particularly true if varying water vapour and nitric acid concentrations are taken into account. However, in some years more PSC than might be expected from examination of simple temperature thresholds applied to Met Office analyses are observed in June. The lack of simultaneous nitric acid measurements means that during periods of denitrification the temperature thresholds significantly overestimate PSC occurrence, this is particularly clear in October in several years.

Inspection of the distribution of temperatures relative to the $T_{\mathrm{NAT}}$ threshold shown in Fig. 8 shows that the latitudinal variation of POAM III measurements means that these values are not representative of the whole vortex, as previously identified by Pitts et al. (2007). However, the POAM III observations occur in regions where temperatures are conducive for gravity wave induced PSC formation. When focusing on the June results examination of the probability of temperatures below the $T_{\mathrm{NAT}}$ threshold based on CHAMP observations show a slightly improved predictive capability compared to the PSC occurrence derived from Met Office data. CHAMP RO observations are consistently close or over-estimate the PSC occurrence measured by POAM III. This makes some physical sense given that these thresholds are a necessary but not sufficient condition for PSC formation. This improved correspondence may partially be associated with a warm bias in the Met Office temperature data compared to CHAMP RO measurements.

Analysis of the CHAMP temperature observations suggests that temperature perturbations associated with gravity waves are likely to be important in explaining the enhanced PSC incidence observed in June. Examination of the longitudinal structure in June 2005 also shows that the enhanced PSC occurrence is close to the Antarctic peninsula, a known mountain wave "hotspot". Analysis also suggests that the temperature perturbations produced by gravity waves are most important early in the Antarctic winter, but have a smaller effect later in the winter. However, it should be noted that the 'enhanced' PSC occurrence in June is likely to be associated with both strong temperature perturbations associated with mountain waves and temperatures hovering slightly above the $T_{\mathrm{NAT}}$ temperature threshold. The lower values later in the winter are likely to be affected by the cooler background temperatures making extra temperature threshold crossings more unlikely and the reduced magnitude of gravity waves perturbations observed as POAM III begins to sample poleward of the peninsula.

Based on Fig. 10 temperature perturbations associated with gravity waves contribute to the formation of about $15 \%$ of the $T_{\mathrm{NAT}}$ threshold crossings observed, a value which corresponds well to the value derived in Innis and Klekociuk (2006). It is important to note that the values derived from CHAMP data may be underestimates because of observational filtering and thus should be considered conservative estimates. Studies which focus on ozone depletion in the early period of the Antarctic winter may need to be aware or parameterize for the effect of gravity waves on PSC formation. In the future we aim to complete a more conclusive study by using measurements from CALIPSO and the COSMIC satellites.

Acknowledgements. We are grateful to the GFZ Potsdam and the ISDC for providing the CHAMP data. The POAM III data is kindly provided at http://wvms.nrl.navy.mil/POAM/data/data.html. AJM would like to acknowledge the support provided by the Brian Mason Scientific and Technical Trust. AJM would also like to thank Rebecca Batchelor and Simon Alexander for useful comments on an early version of this work. We would like to thank 
four anonymous referees for providing suggestions for further analysis and improvements to the text.

Edited by: P. Haynes

\section{References}

Alfred, J., Fromm, M., Bevilacqua, R., Nedoluha, G., Strawa, A., Poole, L., and Wickert, J.: Observations and analysis of polar stratospheric clouds detected by POAM III and SAGE III during the SOLVE II/VINTERSOL campaign in the 2002/2003 Northern Hemisphere winter, Atmos. Chem. Phys., 7, 2151-2163, 2007, http://www.atmos-chem-phys.net/7/2151/2007/.

Baumgaertner, A. J. G. and McDonald, A. J.: A gravity wave climatology for Antarctica compiled from Challenging Minisatellite Payload/Global Positioning System (CHAMP/GPS) radio occultations, J. Geophys. Res.-Atmos., 112, D05103, doi:10.1029/2006JD007504, 2007.

Carslaw, K. S., Luo, B. P., Clegg, S. L., Peter, T., Brimblecombe, P., and Crutzen, P. J.: Stratospheric Aerosol Growth and Hno3 Gas-Phase Depletion from Coupled Hno3 and Water-Uptake by Liquid Particles, Geophys. Res. Lett., 21, 2479-2482, 1994.

Carslaw, K. S., Wirth, M., Tsias, A., Luo, B. P., Dornbrack, A., Leutbecher, M., Volkert, H., Renger, W., Bacmeister, J. T., Reimers, E., and Peter, T. H.: Increased stratospheric ozone depletion due to mountain-induced atmospheric waves, Nature, 391, 675-678, 1998.

de la Torre, A. and Alexander, P.: Gravity waves above Andes detected from GPS radio occultation temperature profiles: Mountain forcing?, Geophys. Res. Lett., 32, L17815, doi:10.1029/2005GL022959, 2005.

Eckermann, S. D., Hoffmann, L., Hopfner, M., Wu, D. L., and Alexander, M. J.: Antarctic NAT PSC belt of June 2003: Observational validation of the mountain wave seeding hypothesis, Geophys. Res. Lett., 36, doi:10.1029/2008GL036629, 2009.

Felton, M. A., Kovacs, T. A., Omar, A. H., and A., H. C.: Classification of Polar Stratospheric Clouds Using LIDAR Measurements From the SAGE III Ozone Loss and Validation Experiment, Tech. Rep. ARL-TR-4154, http://www.arl.army.mil/www/default.cfm?Action=17\&Page= 239\&To\% pic $=$ TechnicalReports\&Year=2007, 2007.

Fromm, M., Alfred, J., and Pitts, M.: A unified, long-term, highlatitude stratospheric aerosol and cloud database using SAM II, SAGE II, and POAM II/III data: Algorithm description, database definition, and climatology, J. Geophys. Res.-Atmos., 108(D12), 14366, doi:10.1029/2002JD002772, 2003.

Fromm, M. D., Lumpe, J. D., Bevilacqua, R. M., Shettle, E. P., Hornstein, J., Massie, S. T., and Fricke, K. H.: Observations of Antarctic polar stratospheric clouds by POAM II: 1994-1996, J. Geophys. Res.-Atmos., 102, 23659-23672, 1997.

Fueglistaler, S. and, B. P. L. B. P., C. Voigt, C., K. S. Carslaw, K. S., and Peter, T.: NAT-rock formation by mother clouds: a microphysical model study, Atmos. Chem. Phys., 2, 93-98, 2002, http://www.atmos-chem-phys.net/2/93/2002/.

Gobiet, A., Foelsche, U., Steiner, A. K., Borsche, M., Kirchengast, G., and Wickert, J.: Climatological validation of stratospheric temperatures in ECMWF operational analyses with CHAMP radio occultation data, Geophys. Res. Lett., 32, L12806, doi:10.1029/2005GL022617, 2005.
Hanson, D. and Mauersberger, K.: Laboratory Studies of the NitricAcid Trihydrate - Implications for the South Polar Stratosphere, Geophys. Res. Lett., 15, 855-858, 1988.

Hertzog, A., Boccara, G., Vincent, R. A., Vial, F., and Cocquerez, P.: Estimation of gravity wave momentum flux and phase speeds from quasi-Lagrangian stratospheric balloon flights. Part II: Results from the Vorcore campaign in Antarctica, J. Atmos. Sci., 65, 3056-3070, 2008.

Hitchman, M. H., Buker, M. L., Tripoli, G. J., Browell, E. V., Grant, W. B., McGee, T. J., and Burris, J. F.: Nonorographic generation of Arctic polar stratospheric clouds during December 1999, J. Geophys. Res.-Atmos., 108(D5), 8325, doi:10.29/2001JD001034, 2003.

Höpfner, M., Blumenstock, T., Hase, F., Zimmermann, A., Flentje, H., and Fueglistaler, S.: Mountain polar stratospheric cloud measurements by ground based FTIR solar absorption spectroscopy, Geophys. Res. Lett., 28, 2189-2192, 2001.

Höpfner, M., Larsen, N., Spang, R., Luo, B. P., Ma, J., Svendsen, S. H., Eckermann, S. D., Knudsen, B., Massoli, P., Cairo, F., Stiller, G., Von Clarmann, T., and Fischer, H.: MIPAS detects Antarctic stratospheric belt of NAT PSCs caused by mountain waves, Atmos. Chem. Phys., 6, 1221-1230, 2006, http://www.atmos-chem-phys.net/6/1221/2006/.

Huck, P. E., McDonald, A. J., Bodeker, G. E., and Struthers, H.: Interannual variability in Antarctic ozone depletion controlled by planetary waves and polar temperature, Geophys. Res. Lett., 32, L13819, doi:10.1029/2005GL022943, 2005.

Innis, J. L. and Klekociuk, A. R.: Planetary wave and gravity wave influence on the occurrence of polar stratospheric clouds over Davis Station, Antarctica, seen in lidar and radiosonde observations, J. Geophys. Res.-Atmos., 111, D22102, doi:10.1029/2006JD007629, 2006.

Irie, H., Pagan, K. L., Tabazadeh, A., Legg, M. J., and Sugita, T.: Investigation of polar stratospheric cloud solid particle formation mechanisms using ILAS and AVHRR observations in the Arctic, Geophys. Res. Lett., 31, L15107, doi:10.1029/2004GL020246, 2004.

Jakob, C. and Tselioudis, G.: Objective identification of cloud regimes in the Tropical Western Pacific, Geophys. Res. Lett., 30(21), 2082, doi:10.1029/2003GL018367, 2003.

Juarez, M. D., Marcus, S., Dornbrack, A., Schroder, T. M., Kivi, R., Iijima, B. A., Hajj, G. A., and Mannucci, A. J.: Detection of temperatures conducive to Arctic polar stratospheric clouds using CHAMP and SAC-C radio occultation data, J. Geophys. Res.-Atmos., 114, D07112, doi:10.1029/2008JD011261, 2009.

Lange, M. and Jacobi, C.: Analysis of gravity waves from radio occultation measurements., in: First CHAMP Mission Results for Gravity, Magnetic and Atmospheric Studies, edited by Reigber, C., Lühr, H., and Schwintzer, P., Springer, Berlin, Germany, 479-484, 2003.

Lorenc, A. C., Ballard, S. P., Bell, R. S., Ingleby, N. B., Andrews, P. L. F., Barker, D. M., Bray, J. R., Clayton, A. M., Dalby, T., Li, D., Payne, T. J., and Saunders, F. W.: The Met. Office global threedimensional variational data assimilation scheme, Q. J. Roy. Meteor. Soc., 126, 2991-3012, 2000.

Lowe, D. and MacKenzie, A. R.: Polar stratospheric cloud microphysics and chemistry, J. Atmos. Solar-Terr. Phys., 70, 13-40, 2008.

Lucke, R. L., Korwan, D. R., Bevilacqua, R. M., Hornstein, J. S., 
Shettle, E. P., Chen, D. T., Daehler, M., Lumpe, J. D., Fromm, M. D., Debrestian, D., Neff, B., Squire, M., Konig-Langlo, G., and Davies, J.: The Polar Ozone and Aerosol Measurement (POAM) III instrument and early validation results, J. Geophys. Res.-Atmos., 104, 18785-18799, 1999.

Luntama, J. P., Kirchengast, G., Borsche, M., Foelsche, U., Steiner, A., Healy, S., von Engeln, A., O'Clerigh, E., and Marquardt, C.: Prospects of the Eps Gras Mission for Operational Atmospheric Applications, Bulletin of the American Meteor. Soc., 89, 18631875, 2008.

Marti, J. and Mauersberger, K.: A Survey and New Measurements of Ice Vapor-Pressure at Temperatures between 170 and $250 \mathrm{k}$, Geophys. Res. Lett., 20, 363-366, 1993.

McDonald, A. J. and Hertzog, A.: Comparison of stratospheric measurements made by CHAMP radio occultation and Strateole/Vorcore in situ data, Geophys. Res. Lett., 35, L11805, doi:10.1029/2008GL033338, 2008.

Mergenthaler, J. L., Kumer, J. B., Roche, A. E., and Massie, S. T.: Distribution of Antarctic polar stratospheric clouds as seen by the CLAES experiment, J. Geophys. Res.-Atmos., 102, 1916119170, 1997.

Nedoluha, G. E., Bevilacqua, R. M., Fromm, M. D., Hoppel, K. W., and Allen, D. R.: POAM measurements of PSCs and water vapor in the 2002 Antarctic vortex, Geophys. Res. Lett., 30(15), 1796, doi:10.1029/2003GL017577, 2003.

Noel, V., Hertzog, A., Chepfer, H., and Winker, D. M.: Polar stratospheric clouds over Antarctica from the CALIPSO spaceborne lidar, J. Geophys. Res.-Atmos., 113, D02205, doi:10.1029/2007JD008616, 2008.

Pagan, K. L., Tabazadeh, A., Drdla, K., Hervig, M. E., Eckermann, S. D., Browell, E. V., Legg, M. J., and Foschi, P. G.: Observational evidence against mountain-wave generation of ice nuclei as a prerequisite for the formation of three solid nitric acid polar stratospheric clouds observed in the Arctic in early December 1999, J. Geophys. Res.-Atmos., 109, D04312, doi:10.1029/2003JD003846, 2004.

Parrondo, M. C., Yela, M., Gil, M., von der Gathen, P., and Ochoa, H.: Mid-winter lower stratosphere temperatures in the Antarctic vortex: comparison between observations and ECMWF and NCEP operational models, Atmos. Chem. Phys., 7, 435-441, 2007, http://www.atmos-chem-phys.net/7/435/2007/.

Peter, T.: Microphysics and heterogeneous chemistry of polar stratospheric clouds, Ann. Rev. Phys. Chem., 48, 785-822, 1997.

Pitts, M. C., Thomason, L. W., Poole, L. R., and Winker, D. M.: Characterization of Polar Stratospheric Clouds with spaceborne lidar: CALIPSO and the 2006 Antarctic season, Atmos. Chem. Phys., 7, 5207-5228, 2007,

http://www.atmos-chem-phys.net/7/5207/2007/.

Poole, L. R. and Pitts, M. C.: Polar Stratospheric Cloud Climatology Based on Stratospheric Aerosol Measurement-Ii Observations from 1978 to 1989, J. Geophys. Res.-Atmos., 99, 1308313089, 1994.

Saitoh, N., Hayashida, S., Sugita, T., Nakajima, H., Yokota, T., and Sasano, Y.: Variation in PSC Occurrence Observed with ILAS-II over the Antarctic in 2003, SOLA, 2, 72-75, 2006.

Santee, M. L., Lambert, A., Read, W. G., Livesey, N. J., Cofield, R. E., Cuddy, D. T., Daffer, W. H., Drouin, B. J., Froidevaux, L., Fuller, R. A., Jarnot, R. F., Knosp, B. W., Manney, G. L., Perun, V. S., Snyder, W. V., Stek, P. C., Thurstans, R. P., Wagner,
P. A., Waters, J. W., Muscari, G., de Zafra, R. L., Dibb, J. E., Fahey, D. W., Popp, P. J., Marcy, T. P., Jucks, K. W., Toon, G. C., Stachnik, R. A., Bernath, P. F., Boone, C. D., Walker, K. A., Urban, J., and Murtagh, D.: Validation of the Aura Microwave Limb Sounder HNO3 measurements, J. Geophys. Res.-Atmos., 112, D24540, doi:10.1029/2007JD008721, 2007.

Shibata, T., Sato, K., Kobayashi, H., Yabuki, M., and Shiobara, M.: Antarctic polar stratospheric clouds under temperature perturbation by nonorographic inertia gravity waves observed by micropulse lidar at Syowa Station, J. Geophys. Res.-Atmos., 108(D3), 4105, doi:10.1029/2002JD002 713, 2003.

Solomon, S., Garcia, R. R., Rowland, F. S., and Wuebbles, D. J.: On the Depletion of Antarctic Ozone, Nature, 321, 755-758, 1986.

Strawa, A. W., Drdla, K., Fromm, M., Pueschel, R. F., Hoppel, K. W., Browell, E. V., Hamill, P., and Dempsey, D. P.: Discriminating types $\mathrm{Ia}$ and $\mathrm{Ib}$ polar stratospheric clouds in POAM satellite data, J. Geophys. Res.-Atmos., 107(D20), 8291, doi:10.1029/2001JD000 458, 2002.

Svendsen, S. H., Larsen, N., Knudsen, B., Eckermann, S. D., and Browell, E. V.: Influence of mountain waves and NAT nucleation mechanisms on polar stratospheric cloud formation at local and synoptic scales during the 1999-2000 Arctic winter, Atmos. Chem. Phys., 5, 739-753, 2005,

http://www.atmos-chem-phys.net/5/739/2005/.

Tabazadeh, A., Jensen, E. J., Toon, O. B., Drdla, K., and Schoeberl, M. R.: Role of the stratospheric polar freezing belt in denitrification, Science, 291, 2591-2594, 2001.

Teitelbaum, H., Moustaoui, M., and Fromm, M.: Exploring polar stratospheric cloud and ozone minihole formation: The primary importance of synoptic-scale flow perturbations, J. Geophys. Res.-Atmos., 106, 28173-28188, 2001.

Tsias, A., Wirth, M., Carslaw, K. S., Biele, J., Mehrtens, H., Reichardt, J., Wedekind, C., Weiss, V., Renger, W., Neuber, R., von Zahn, U., Stein, B., Santacesaria, V., Stefanutti, L., Fierli, F., Bacmeister, J., and Peter, T.: Aircraft lidar observations of an enhanced type Ia polar stratospheric clouds during APEPOLECAT, J. Geophys. Res.-Atmos., 104, 23961-23969, 1999.

Voigt, C., Schlager, H., Luo, B. P., Dornbrack, A. D., Roiger, A., Stock, P., Curtius, J., Vossing, H., Borrmann, S., Davies, S., Konopka, P., Schiller, C., Shur, G., and Peter, T.: Nitric Acid Trihydrate (NAT) formation at low NAT supersaturation in Polar Stratospheric Clouds (PSCs), Atmos. Chem. Phys., 5, 13711380, 2005, http://www.atmos-chem-phys.net/5/1371/2005/.

Wang, K. Y. and Lin, S. C.: First continuous GPS soundings of temperature structure over Antarctic winter from FORMOSAT3/COSMIC constellation, Geophys. Res. Lett., 34, L12805, doi:10.1029/2007GL030159, 2007.

Wang, Z., Stephens, G., Deshler, T., Trepte, C., Parish, T., Vane, D., Winker, D., Liu, D., and Adhikari, L.: Association of Antarctic polar stratospheric cloud formation on tropospheric cloud systems, Geophys. Res. Lett., 35, L13806, doi:10.1029/2008GL034 209, 2008.

Wickert, J., Schmidt, T., Beyerle, G., Konig, R., and Reigber, C.: The radio occultation experiment aboard CHAMP: Operational data analysis and validation of vertical atmospheric profiles, J. Meteor. Soc. Jpn., 82, 381-395, 2004.

World Meteorological Organization: Scientific Assessment of Ozone Depletion: 2006, Global Ozone Research and Monitoring Project - Report No. 50, 572 pp., Geneva, Switzerland, 2007. 
Wu, D. L. and Jiang, J. H.: MLS observations of atmospheric gravity waves over Antarctica, J. Geophys. Res.-Atmos., 107(D24), 4773, doi:10.1029/2002JD002390, 2002. 